\title{
Numerical methods for parameter identification in a convection-diffusion equation
}

\author{
T. S. Shores*
}

(Received 8 August 2003; revised 30 January 2004)

\begin{abstract}
We use a recently developed Sinc-Galerkin method for the solution of non-self-adjoint equations to solve a parameter identification problem arising from the one-dimensional convection-diffusion equation. This method is well suited for unbounded domains and certain singularities in the coefficients, as we illustrate by several examples. Practical aspects of implementation of this method are considered in detail.
\end{abstract}

\section{Contents}

\section{Introduction}

* Deptartment of Mathematics, University of Nebraska-Lincoln, U.S.A. mailto:tshores@math .unl.edu

See http://anziamj.austms.org.au/V45/CTAC2003/Shor/home.html for this article, (c) Austral. Mathematical Soc. 2004. Published July 18, 2004. ISSN 1446-8735 
2 Algorithm for the direct problem

C662

2.1 Sinc background . . . . . . . . . . . . . . . . C662

2.2 Variational and numerical variational forms . . . . . . C664

3 Algorithm for the inverse problem

C665

4 Examples

C667

4.1 Example . . . . . . . . . . . . . . . . . C668

4.2 Example . . . . . . . . . . . . . . . . . . C668

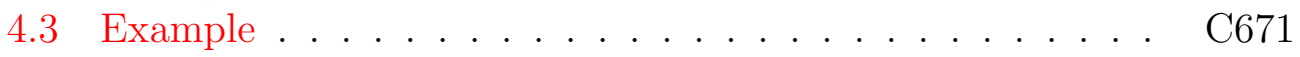

5 Conclusions

C672

References

C674

\section{Introduction}

Sinc methods for the numerical solution of ordinary differential equations have been found to be a very effective technique, particularly for problems with singular solutions and those on unbounded domains. In this paper we consider an inverse problem associated with the direct problem of solving a steady state convection-diffusion system in one space dimension given by

$$
\begin{aligned}
L c \equiv-\left(D(x) c_{x}\right)_{x}+(v(x) c)_{x}+\lambda(x) c & =f(x), \quad x>0, \\
A c(0)+B c_{x}(0) & =G, \quad B \neq 0 \\
c(\infty) & =0 .
\end{aligned}
$$

The inverse problem is that of determining the diffusion coefficient $D(x)$ from measurements of the solution $c(x)$. This direct problem is singular since it is defined on a semi-infinite interval. Moreover, we allow certain singularities in the coefficients $v(x), \lambda(x)$ at the endpoints. We make assumptions about the coefficients and the solution as well. In particular, we assume that the 
dispersion coefficient $D(x)$ is continuous, bounded and tends to a constant as $x \rightarrow \infty$. These conditions ensure well-posedness of the direct problem. Additional conditions are required for Sinc methods to work and we discuss these in Section 3.

There are many Sinc approaches to solving second order differential equations (see [4, 6, e.g.] for comprehensive surveys). These typically require a unit coefficient for the second order term of the differential equation, which would necessitate differentiation of the diffusion coefficient $D(x)$. Methods that avoid this differentiation were developed in [2, 4, p.188]) for selfadjoint problems on a finite interval with Dirichlet boundary conditions. The direct solver that we employ was developed in [5] to also avoid differentiation of the diffusion coefficient $D(x)$ and to handle the case of a non-selfadjoint problem with mixed boundary conditions as well. We shall show that when properly tuned, it yields a robust and effective tool for identification of $D(x)$. Electronic copies of the programs used in this paper are available upon request from the author. These programs were written in the language Octave [1] but are easily ported to MATLAB.

\section{Algorithm for the direct problem}

\subsection{Sinc background}

Recall that the sinc function is defined for all $z \in \mathbb{C}$ by

$$
\operatorname{sinc}(z)= \begin{cases}\frac{\sin (\pi z)}{\pi z}, & z \neq 0 \\ 1, & z=0\end{cases}
$$

Let $h$ be a positive constant. We denote the sinc basis functions by

$$
S(k, h)(x)=\operatorname{sinc}\left(\frac{x-k h}{h}\right), \quad k \in \mathbb{Z}, \quad-\infty<x<\infty .
$$


The sinc differentiation operator matrix of dimension $m+1$, is given by $I^{(1)}=\left[\delta_{j k}^{(1)}\right]_{j, k=0}^{m}$, where $\left.\delta_{j k}^{(1)} \equiv h S^{\prime}(j, h)(x)\right|_{x=x_{k}}$ and similarly $I=I^{(0)}=$ $\left[\delta_{j k}\right]=[S(k, h)(j h)]$.

Let $\phi$ be a conformal map which maps the horizontal strip of radius $d$ about the $x$-axis onto the domain $\mathcal{D}$ with boundary $\Gamma$ and inverse map $\psi$ and $F(z)$ an element of

$$
B(\mathcal{D})=\left\{F(z) \mid F \text { is analytic on } D, \int_{\Gamma}|F(w) d w|<\infty\right\}
$$

Assume $F(x)$ is real valued on $(0, \infty), \gamma$ is analytic, $\phi^{\prime} F / \gamma \in B(\mathcal{D})$ and suppose there are positive constants $\alpha, \beta$ and $C$ so that

$$
\left|\frac{F(\xi)}{\gamma(\xi)}\right| \leq C \begin{cases}\exp (-\alpha|\phi(\xi)|), & \xi \in \Gamma_{a} \\ \exp (-\beta|\phi(\xi)|), & \xi \in \Gamma_{b}\end{cases}
$$

where $\Gamma_{a} \equiv\{\xi \in \Gamma \mid \phi(\xi)=x \in(-\infty, 0)\}$ and $\Gamma_{b} \equiv\{\xi \in \Gamma \mid \phi(\xi)=x \in$ $[0, \infty)\}$.

Theorem 1 Assume notation as above and make parameter selections

$$
N=\left\lceil\frac{\alpha}{\beta} M\right\rceil \quad \text { and } \quad h=\left(\frac{\pi d}{\alpha M}\right)^{\frac{1}{2}} \leq \frac{2 \pi d}{\ln (2)} .
$$

Set $w_{k}=\psi(k h)$. Then there exists constants $K, L$ independent of $M$ and $m=0,1, \ldots, n$, such that for all $\xi \in \Gamma$

$$
\left\|\frac{d^{m}}{d \xi^{m}} F(\xi)-\sum_{k=-M}^{N} \frac{F\left(w_{k}\right)}{g\left(w_{k}\right)} \frac{d^{m}}{d \xi^{m}} \gamma(\xi) S(k, h) \circ \phi(\xi)\right\|_{\infty} \leq K M^{(m+1) / 2} e^{-\sqrt{\pi d \alpha M}}
$$

and

$$
\left|\int_{a}^{b}(v w[S(j, h) \circ \phi])(x) d x-h \frac{v w}{\phi^{\prime}}\left(x_{j}\right)\right| \leq L M e^{-\sqrt{\pi d \alpha M}} .
$$


Proofs of these theorems can be found in the Stenger text [6] or the Lund and Bowers text [4]. This fact exhibits the exponential accuracy of correctly tuned sinc approximations. It also underscores the need to understand the asymptotic nature of the function to be approximated. Extensive discussions of these matters can be found in the texts cited.

\subsection{Variational and numerical variational forms}

Following [5], we multiply the differential equation of system (1) by suitable test functions $u$ which vanish at the endpoints, and use integration by parts to obtain

$$
\int_{0}^{\infty}\left(D c_{x} u_{x}-v c u_{x}+\lambda c u\right) d x=\int_{0}^{\infty} f(x) u d x .
$$

We can express this variational form as $T(c, u)=R(u)$. Now define

$$
\tilde{c}(x) \equiv c(x)-c^{\prime}(0) q_{0}(x)-c(0) q_{1}(x)
$$

for suitable cardinal functions $q_{0}(x), q_{1}(x)$ satisfying

$$
q_{0}(0)=0, \quad q_{1}(0)=1, \quad q_{0}^{\prime}(0)=1, \quad q_{1}^{\prime}(0)=0,
$$

and we obtain that $\tilde{c}(0)=0$ and $\tilde{c}^{\prime}(0)=0$. Sinc approximation theory can be applied to this function and it yields the numerical variational form

$$
T(\tilde{c}, u)+c_{x}(0) T\left(q_{0}, u\right)+c(0) T\left(q_{1}, u\right)=R(u) .
$$

For a Sinc-Galerkin approximation we choose test functions $u_{j}(x)=$ $\gamma(x) S(j, h) \circ \phi(x)$ where $\gamma(x)$ is a weight function and $\phi(x)$ is a conformal map from $(0, \infty)$ to $(-\infty, \infty)$, such as $\phi(x)=\ln (x)$. We assume that the coefficients of the problem (1) and the choice of cardinal functions $q_{0}$ and $q_{1}$ are such that the solution $\tilde{c}(x)$ satisfies the restrictions of Theorem 1. Thus we can approximate $\tilde{c}$ exponentially well by

$$
\tilde{c}_{m}(x)=\sum_{j=-M_{x}}^{N_{x}} \frac{d_{j}}{\gamma\left(w_{j}\right)} \gamma(x) S(j, h) \circ \phi(x),
$$


where $\gamma$ is an appropriately chosen so that $\tilde{c}^{\prime}(x)$ is accurately approximated by the derivative of the cardinal sum and $m=M_{x}+N_{x}+1$, the number of sinc nodes. More importantly, this formula can be differentiated to obtain an exponentially accurate formula for the derivative of $\tilde{c}^{\prime}(x)$. This formula can be inserted into the numerical variational form for the direct problem.

Let $d=\left[d_{j}\right]_{j=1}^{m}$ be the the vector of sinc node values of $\tilde{c}_{m}(x)$. The discrete system corresponding to the numerical variational form can, after much algebraic manipulation, be expressed as a linear system with coefficient matrix $M$, namely

$$
M d+c_{0}^{\prime} T_{0}+c_{0} T_{1}=R_{\mathrm{dis}},
$$

where $c_{0}=c(0)$ and $c_{0}^{\prime}=c_{x}(0)$. Let $w_{s}=M^{-1} R_{\text {dis }}, w_{0} \equiv M^{-1}\left(T_{0}\right)$ and $w_{1} \equiv M^{-1} T_{1}$ and we may write this equation in the form

$$
d=w_{s}-c_{0}^{\prime} w_{0}-c_{0} w_{1} .
$$

A scheme for computing $c_{0}^{\prime}$ and $c_{0}$ can be derived by integrating the differential equation of the problem from 0 to $\infty$ to obtain

$$
\int_{0}^{\infty}(f(x)-\lambda(x) c) d x=D(0) c_{x}(0)-v(0) c(0) .
$$

This equation can be discretized to give one more linear equation in $c_{0}^{\prime}$ and $c_{0}$ in addition to the left boundary condition,

$$
A c_{0}+B c_{0}^{\prime}=G .
$$

For details we refer the reader to [5].

\section{Algorithm for the inverse problem}

The problem we consider is as follows: Given a sampling of the solution $c(x)$ of the system (1) at $n$ various points (possibly with noise) and all parameters except $D(x)$, to recover an approximation to $D(x)$. 
Suppose that $c(x)$ solves the system $L c=f$ together with the boundary conditions. In the setting of an inverse problem, we are usually given the solution $c(x)$ by point-wise measurements $d$, which may have noise as well. If the operator $H$ represents point-wise evaluation, then the appropriate operator for inversion is

$$
F(D)=d=H c=H L^{-1}(f),
$$

and we want to solve $F(D)=d$ for the parameter $D$. We assume the direct problem is well posed, so that $F$ is well defined. As usual, "inverting" $F$ is an ill-posed problem, so we resort to the standard Tikhonov regularization technique and cast the problem in the form:

Minimize the functional

$$
T_{\alpha}(D)=\frac{1}{2}\|F(D)-d\|^{2}+\alpha\|P(D)\|^{2} .
$$

We now proceed to discretize this operator $T_{\alpha}$. The semi-infinite interval $(0, \infty)$ poses special difficulties, since the coordinates of the first and second term of the Tikhonov functional are quite different in nature.

Penalty Term $P(D)$ : We choose to define the penalty function in terms of the defining constituents of a solution of the direct problem and a possible nonzero value at infinity. Thus our full model for $D(x)$ (in analogy with the solution $c(x)$ of the direct problem) is

$$
D(x)=\widetilde{D}(x)+D^{\prime}(0) q_{0}(x)+D(0) q_{1}(x)+D(\infty) q_{2}(x),
$$

where $q_{0}, q_{1}$ are as above, and $q_{2}(0)=0, q_{2}^{\prime}(0)=0, q_{2}(\infty)=1$. As in Section 2.2, the cardinal functions are chosen in such a way that Theorem 1 applies to the reduced function $\widetilde{D}(x)$. This amounts to an analyticity restriction on $D(z)$ in the domain $\mathcal{D}$ (which includes the positive $x$-axis) of Theorem 1. Hence we define the penalty function

$$
P(D)^{2}=a_{1}\left|D^{\prime}(0)\right|^{2}+a_{2}|D(0)|^{2}+a_{3}|D(\infty)|^{2}+a_{4}\|\widetilde{D}\|^{2}+a_{5}\left\|\widetilde{D}^{\prime}\right\|^{2} .
$$


This function discretizes very nicely and Sinc theory can be used to approximate the last two terms exponentially accurately, thanks to our assumptions about $D$. In practice, the coefficient of $D(\infty)$ tends to be unimportant relative to the others, except for the fact that it tends to overly damp the solution when larger values of the regularization parameter $\alpha$. Hence, in the absence of additional information, we typically take all coefficients to be 1 except $a_{3}$, which is chosen to be small, say $a_{3}=0.001$.

Functional Term: The term $\frac{1}{2}\|F(D)-d\|^{2}$ is more problematic since the sampled values,

$$
d \approx\left[c\left(x_{k}\right)\right]_{k=1}^{n},
$$

are not necessarily at the sinc nodes (in fact, almost certainly not), yet in the limit this term should provide global information about $D$ itself. We choose the simplest possible interpretation: that $F(D)-d$ is a fixed vector of values and we simply use the Euclidean norm (scaled by $1 / \sqrt{n}$, where $n$ is the dimension of the vector so as to approximate integrals in the limit of $n$ ) on this part of the Tikhonov functional.

Discretization is now very straightforward: Approximate $L^{-1}(f)$ on a sinc grid, then use a projection matrix $H$ to evaluate the approximate sinc solution at the sampling nodes. Thus, if there are $m$ sinc nodes and $n$ sampling nodes, $L^{-1}(f)$ is discretized to a vector $v=\left[v_{j}\right]_{j=1}^{m}$ and the discrete version of $F(D)-d$ is

$$
F_{n}(D)-d=H v-d
$$

\section{Examples}

We shall illustrate the method by several examples. These examples are somewhat artificial in the sense that the exact answer is known in advance and is even used to generate parameters of the problem. Nonetheless, such 
TABle 1: Accuracy of Sinc approximation for Example 4.1.

\begin{tabular}{|c|cccc|}
\hline & \multicolumn{4}{|c|}{$\alpha=1, \beta=1, d=\pi / 3$} \\
$M_{x}$ & $m$ & $h$ & $|e(0)|$ & $\left\|e\left(x_{k}\right)\right\|_{\infty}$ \\
\hline 16 & 22 & 0.45 & $1.75 \mathrm{e}-03$ & $3.39 \mathrm{e}-03$ \\
32 & 40 & 0.32 & $4.89 \mathrm{e}-04$ & $5.04 \mathrm{e}-04$ \\
64 & 78 & 0.22 & $2.27 \mathrm{e}-05$ & $2.78 \mathrm{e}-05$ \\
128 & 148 & 0.16 & $1.69 \mathrm{e}-08$ & $1.28 \mathrm{e}-06$ \\
\hline
\end{tabular}

an approach is needed to simulate the generation of data and to evaluate the accuracy of the algorithm.

\subsection{Example}

The direct algorithm is illustrated by the following example, which is taken from [5]. Consider the steady-state convection-diffusion problem

$$
\begin{aligned}
-\left(\left(1-0.5 e^{-2 x}\right) c_{x}\right)_{x}+c_{x}+c & =f(x), \quad x>0, \\
c(0)-0.5 c_{x}(0) & =1, \\
c(\infty) & =0 .
\end{aligned}
$$

Here the solution that generated the problem is $c(x)=\exp \left(-x^{2} / 4\right)$. Some results appear in Table 1. Similar results are found over a range of examples. The parameters $\alpha, \beta, d$ play the same role as Theorem 1. For most inverse problems that we have considered, a sinc system of order $m$ between $m=40$ and $m=80$ suffices.

\subsection{Example}

Consider the direct problem which constitutes Example 4.1. Suppose that we are given a sampling of the true solution at $n$ equally spaced nodes (equal 
TABle 2: Computations for Example 4.2.

\begin{tabular}{|cccc|cccc|}
\hline$n$ & BFGS & Error & $\alpha$ & $m$ (10\% noise) & BFGS & Error & $\alpha$ \\
\hline 4 & 95 & 0.01 & 0.00048 & 4 & 74 & 0.02 & 0.00083 \\
20 & 113 & 0.0047 & 0.00022 & 20 & 96 & 0.014 & 0.001 \\
40 & 108 & 0.0047 & 0.00022 & 40 & 90 & 0.038 & 0.00083 \\
\hline
\end{tabular}

spacing is not a requirement but a convenience here). Take as starting point the constant function $D_{0}=2$ and sinc system size $m=40$. We sample the true solution both with and without noise at evenly spaced points across the interval $[0.2,2.4]$. The reason for this choice is that one can see on examination of computed solutions that the solution vanishes very rapidly. In practical measurements one would expect error to swamp very small values; hence it is preferable to sample in regions where the forward solution is clearly nonzero. In the case of noise we assume a relative error of at most $10 \%$ due to a uniform distribution.

The number of variables in the Tikhonov functional varied from 87 to 123 in this example. A modified Newton BFGS method with Armijo line search applied to the Tikhonov functional proved to be reasonably effective and avoided the expense of full Hessian calculations. The results of our calculations are displayed in Table 2 for cases of exact data and data with of a uniformly distributed random (relative) error of at most 10\%. The number of iterations is listed in the BFGS column. The error displayed is the direct error, that is, the approximate $L_{2}$ norm of $c(x)-c_{\text {approx }}(x)$, where $c_{\text {approx }}(x)$ is the solution to the direct problem obtained by using the computed value of the parameter $D(x)$ in the direct problem and $c(x)$ is the exact solution. Sinc quadrature is used to estimate this norm. Also listed is the choice of regularization parameter suggested by $L$-curve analysis. Note that $c_{\text {approx }}(x)$ is reasonably close to $c(x)$. Of course, closeness of $c_{\text {approx }}(x)$ to $c(x)$ is no guarantee that the computed value $D_{\text {approx }}(x)$ will be similarly close to the exact diffusion coefficient $D(x)$. Graphs of exact and computed values for the parameter $D(x)$ are presented in Figure 1. 


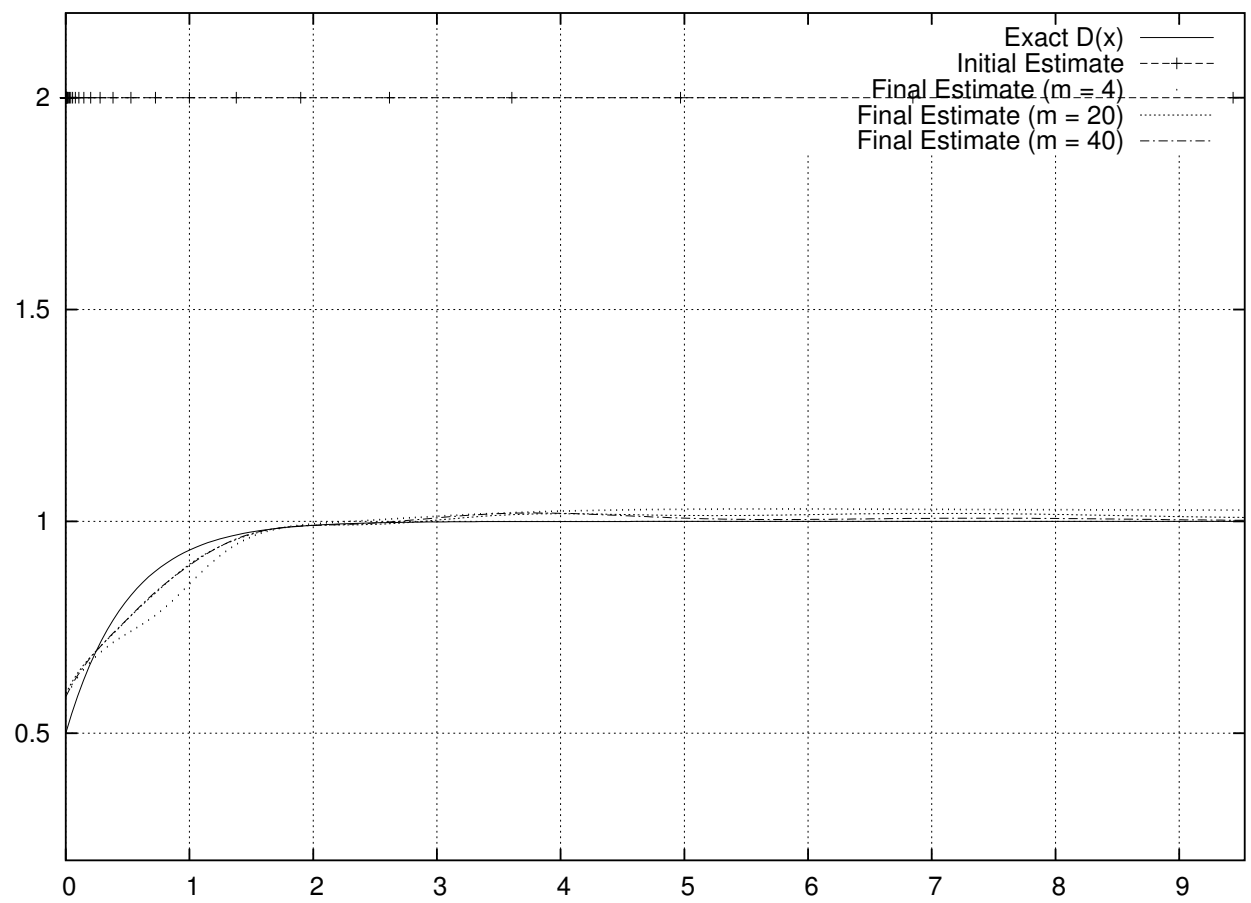

Figure 1: Approximations to parameter $D(x)$ in Example 4.2. 
The choice of regularization parameter $\alpha$ is a complex issue which is complicated by the fact that our problem is non-linear. In these examples we used the $L$-curve approach. However, we use the penalty functional $J(D)$ in place of $\|D\|$ since the latter term is not even defined in the traditional sense, while $J(D)$ serves as a substitute for a norm. It is well known that the $L$ curve selection method need not be a convergent strategy (see Vogel [7]) even for linear inverse problems. We use the $L$-curve as a tool to help us achieve a balance between regularization and residual terms. Therefore, irregularities in the behavior of the $L$-curve are not a matter of special concern. In practice, the $L$-curve seems to be a very effective tool. For a detailed analysis of various strategies for choice of a regularization parameter, see Kilmer and O'Leary [3, e.g.] or the text [8] by Vogel. We conservatively choose the largest $\alpha$ at which a significant corner occurs. In most cases this is graphically obvious, although in a few cases the $L$-curve was so smooth that a "corner" was difficult to identify. In such cases we approximated the curvature numerically and selected the point of maximum numerical curvature. In a few other cases the $L$-curve was erratic and several choices for a "corner" were possible.

\subsection{Example}

Our final steady-state convection-diffusion problem exhibits singularities in both the coefficients and the solution $c(x)=x^{3 / 2} e^{-x}$ which is used to generate the right hand side function in the system

$$
\begin{aligned}
-c_{x x}+c_{x}+\frac{3}{4 x^{2}} c & =f(x), \quad x>0, \\
c(0)-c_{x}(0) & =0 \\
c(\infty) & =0 .
\end{aligned}
$$

The singularities make the problem somewhat more intractable than the previous example. In this problem fairly conservative choices of the sinc parameters $\alpha=1, \beta=1, d=\pi / 3$ (the same as in Example 4.1) work 
TABle 3: Computations for Example 4.3.

\begin{tabular}{|cccc|cccc|}
\hline$m$ & BFGS & Error & $\alpha$ & $m$ (10\% noise) & BFGS & Error & $\alpha$ \\
\hline 4 & 93 & 0.12 & 0.00022 & 4 & 115 & 0.13 & 0.00022 \\
20 & 103 & 0.0024 & 0.00022 & 20 & 118 & 0.013 & 0.0004 \\
40 & 95 & 0.0028 & 0.00022 & 40 & 85 & 0.035 & 0.00091 \\
\hline
\end{tabular}

TABle 4: Computations for Example 4.3 with sampling at sinc nodes.

\begin{tabular}{|cccc|cccc|}
\hline$m$ & BFGS & Error & $\alpha$ & $m$ (10\% noise) & BFGS & Error & $\alpha$ \\
\hline 20 & 103 & 0.0095 & 0.00022 & 20 & 108 & 0.027 & 0.0005 \\
40 & 97 & 0.0138 & 0.00022 & 40 & 111 & 0.060 & 0.00083 \\
\hline
\end{tabular}

reasonably well. Our results are summarized in Table 3 and Figure 2 for the same types of error and measurements as in Example 4.2.

A natural speculation about these examples is that sinc nodes might yield better results than the equally spaced nodes used in Example 4.2 and 4.3. To compare results we revisit Example 4.2 and replace equally spaced nodes by sinc nodes. In order for the comparison to be fair, we must sample no more nodes than we do in the equally spaced case. The number of sample points $n$ is set equal to the number of sinc nodes $m$, which constrains the possibilities for $n$. The choices $n=m=20$ and $n=m=40$ can be generated by taking $M_{x}=15$ and $M_{x}=32$, respectively. A table of the resulting calculations are given in Table 4. A comparison of Tables 3 and 4 shows that sampling at the sinc nodes need not be an optimal strategy.

\section{Conclusions}

The foregoing algorithm and experiments suggest several conclusions:

1. Sinc methods provide a useful tool for solving certain direct singular 


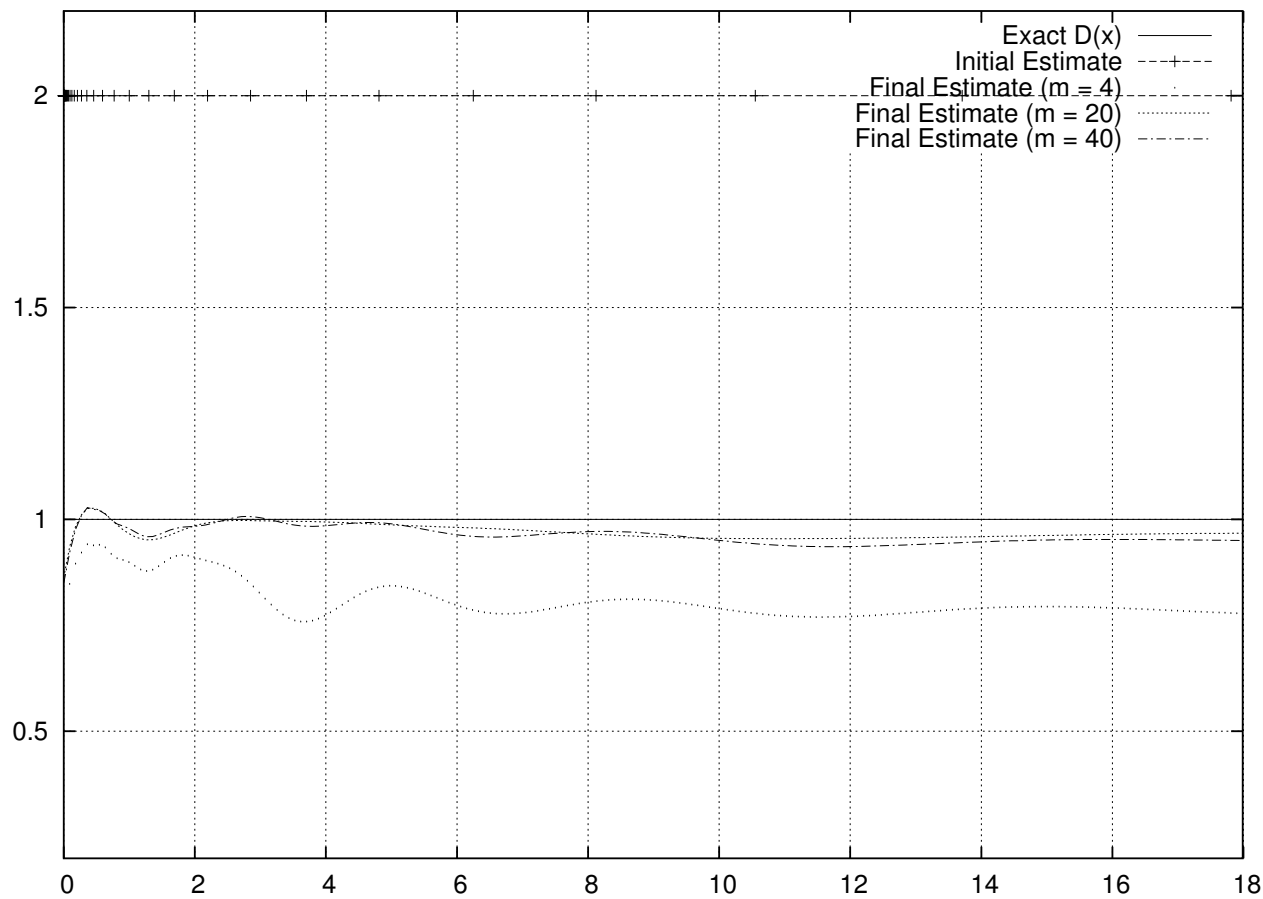

Figure 2: Approximations to parameter $D(x)$ in Example 4.3. 
problems with high accuracy. These solutions can even exhibit mild derivative singularities at the endpoints of the domain of the forward solution such as $c(x)=x^{1 / 2} e^{-x}$ or have singular coefficients such as $\lambda(x)=\frac{3}{4} x^{2}$.

2. Since high accuracy is obtained with relatively small discretization numbers, minimizers used in solving inverse problems end up working with relatively low dimensional systems.

3. The method is fairly robust with respect to noise, provided that the sinc parameters have been carefully chosen for the forward solver and a suitable choice of regularization parameter $\alpha$ is made.

4. The algorithms we have outlined may prove to be useful in the design of real experiments. For example, we saw that placement of sampling nodes impacts upon the quality of results. Further investigations may suggest optimal placements. Also, the issue of uncertainty in measurement warrants further study. Our examples suggest that more data points in the presence of measurement error may not be better. One possibility is to use fewer nodes with repeated measurements at each node.

\section{References}

[1] John W. Eaton. GNU Octave Manual. Network Theory Limited, 2002. C662

[2] J. Lund K. M. McArthur, R. C. Smith and K. L. Bowers. The sinc-galerkin method for parameter dependent self-adjoint problems. Inverse Problems, 6:205-217, 1990. C662 
[3] M. Kilmer and D. O'Leary. Choosing regularization parameters in iterative methods for ill-posed problems. SIAM J. on Matrix Anal. and Applic., 22:1204-1221, 2001. C671

[4] J. Lund and K. Bowers. Sinc Methods for Quadrature and Differential Equations. SIAM, Philadelphia, 1992. C662, C664

[5] J. L. Mueller and T. S. Shores. A new sinc-galerkin method for convection-diffusion equations with mixed boundary conditions. Comput. Math. Appl., 2003. to appear. C662, C664, C665, C668

[6] F. Stenger. Numerical Methods Based on Sinc and Analytic Functions. Springer-Verlag, New York, 1993. C662, C664

[7] C. Vogel. Non-convergence of the l-curve regularization parameter selection method. Inverse Problems, 13:289-303, 1997. C671

[8] C. Vogel. Computational Methods for Inverse Problems. SIAM, Philadelphia, 2002. C671 